\title{
Excess mortality among the elderly in European countries, December 2014 to February 2015
}

K Mølbak (krm@ssi.dk) ${ }^{1}$, L Espenhain ${ }^{1}$, J Nielsen ${ }^{1}$, K Tersago², N Bossuyt², G Denissov³, A Baburin ${ }^{3}$, M Virtanen ${ }^{4}$, A Fouillet ${ }^{5}$, T

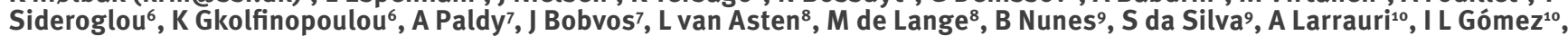

A Tsoumanis ${ }^{11}$, C Junker ${ }^{12}$, H Green ${ }^{13}$, R Pebody ${ }^{13}$, J McMenamin ${ }^{14}$, A Reynolds ${ }^{14}$, A Mazick ${ }^{1}$

1. Statens Serum Institut, Copenhagen, Denmark

2. Scientific Institute of Public Health, Brussels, Belgium

3. National Institute for Health Devlopment, Tallinn, Estonia

4. National Institute for Health and Welfare, Helsinki, Finland

5. French Institute for Public Health Surveillance (Institut de Veille Sanitaire, InVS), Saint-Maurice, France

6. Hellenic Centre for Disease Control and Prevention, Athens, Greece

7. National Institute of Environmental Health, Budapest, Hungary

8. National Institute of Public Health and The Environment (RIVM), the Netherlands

9. Instituto Nacional de Saúde Dr. Ricardo Jorge, Lisbon, Portugal

10. National Centre of Epidemiology; CIBER Epidemiología y Salud Pública (CIBERESP) Institute of Health Carlos III, Madrid, Spain

11. The Public Health Agency of Sweden, Stockholm, Sweden

12. Federal Statistical Office, Neuchâtel, Switzerland

13. Public Health England, London, United Kingdom

14. Health Protection Scotland, Glasgow, United Kingdom

Citation style for this article:

Mølbak K, Espenhain L, Nielsen J, Tersago K, Bossuyt N, Denissov G, Baburin A, Virtanen M, Fouillet A, Sideroglou T, Gkolfinopoulou K, Paldy A, Bobvos J, van Asten L, de Lange M, Nunes B, da Silva S, Larrauri A, Gómez IL, Tsoumanis A, Junker C, Green H, Pebody R, McMenamin J, Reynolds A, Mazick A. Excess mortality among the elderly in European countries, December 2014 to February 2015. Euro Surveill. 2015;20(11): pii=21065. Available online: http://www.eurosurveillance.org/ ViewArticle.aspx?Articleld $=21065$

Article submitted on 23 February 2015 / published on 19 March 2015

Since December 2014 and up to February 2015, the weekly number of excess deaths from all-causes among individuals $\geq 65$ years of age in 14 European countries have been significantly higher than in the four previous winter seasons. The rise in unspecified excess mortality coincides with increased proportion of influenza detection in the European influenza surveillance schemes with a main predominance of influenza $\mathrm{A}\left(\mathrm{H}_{3} \mathrm{~N}_{2}\right)$ viruses seen throughout Europe in the current season, though cold snaps and other respiratory infections may also have had an effect.

In temperate countries in the northern hemisphere, the weekly number of deaths among the elderly (individuals aged $\geq 65$ years) frequently exhibits sharp increases above normal expected levels of mortality during the winter season. The extent of this excess mortality varies considerably between years and between countries. This excess mortality in the elderly is often attributed to seasonal influenza, especially in seasons dominated by influenza $A\left(\mathrm{H}_{3} \mathrm{~N}_{2}\right)$, but factors other than influenza including other respiratory tract infections or environmental conditions (e.g. cold spells) can also play an important contributory role $[1,2]$.

The European monitoring of excess mortality for public health action (EuroMOMO) network (www.euromomo. eu) monitors weekly 'real-time' all-cause age-specific excess mortality in countries in Europe through a standardised approach, allowing pooling of results. In the current winter of $2014 / 15$, there has been an increased number of excess deaths observed among the elderly. We describe this observed excess mortality in Europe using all-cause mortality data up to and including week 9, 2015. The contributions of influenza and other factors to the excess mortality are also considered.

\section{Analyses of all-cause mortality in Europe}

Country level analyses

On a weekly basis, partners in EuroMOMO collect data on the number of deaths from all causes, and undertake timely data analyses by the use of a common algorithm. A time-series Poisson regression is used to predict the number of weekly deaths, adjusted for a linear trend and seasonal variation. The algorithm also corrects for any reporting delay, i.e. delays in the time between date of death and the date the death is registered, which can be lengthy [3].

The main indicators generated are: (i) the total weekly number of all deaths corrected for reporting delay in registration; (ii) the expected weekly number of deaths (baseline); (iii) the weekly number of excess deaths (defined as observed number minus the expected number of deaths); (iv) the standard deviation around the baseline (z-score); and (v) the cumulated total mortality (all age groups) and stratified by age groups ( $<5$, 5-14, 15-64 and $\geq 65$ years).

For this study, sixteen European countries, namely, Belgium, Denmark, Estonia, Finland, France, Greece (municipalities of Athens, Keratsini-Drapetsona and Pireas and prefectures of Magnisia, Sporades, Kerkira, Achaia, Kavala and Thasos), Hungary, the Netherlands, Portugal, Spain, Sweden, Switzerland and the United 
Kingdom (UK: England, Northern Ireland, Wales, and Scotland) provided data for all-cause mortality analyses at country level.

\section{Pooled country data analyses}

Although sixteen countries submitted data to EuroMOMO, partners with marked delays in receiving data were not included in the pooled analyses. This resulted in data from 14 countries (Belgium, Denmark, Estonia, Finland, France, Hungary, the Netherlands, Portugal, Spain, Sweden, Switzerland and the UK (England, Wales, and Scotland)) being included for such analyses. We used the stratified method as previously described [4]. The time-series in the pooled analysis included data from week 23, 2010 to week 9, 2015.

\section{Comparison of mortality patterns}

We used z-scores to standardise outputs enabling comparison of mortality patterns between different countries and between different time-periods. Excess mortality above two z-scores from the baseline in two consecutive weeks, was defined as above the normal level of the standard variation of data. The EuroMOMO hub, situated at Statens Serum Institut in Denmark, compiled the data outputs from individual partners.
Analyses in an epidemiological context

We collected information on influenza activity from influenza activity maps at the European Centre for Disease Prevention and Control (ECDC)'s website [5,6] and the joint ECDC/World Health Organization (WHO) Regional Office for Europe bulletin, FluNews Europe [7]. This included weekly indicators of intensity of transmission on influenza-like illness and proportion of positive influenza virus detections in the sentinel surveillance schemes in European countries. Finally, EuroMOMO network participants provided information on the epidemiological situation in the respective contributing countries, including temperature data.

\section{Results}

The country-specific analyses showed that in the present winter of $2014 / 15$, all-cause excess mortality among the elderly has been above two z-scores from the baseline, for two consecutive weeks or more, in Belgium, France, Greece, Hungary, the Netherlands, Portugal, Spain, Switzerland, Sweden, England, Northern Ireland, Scotland and Wales. Mortality started to increase between week 50 and 52 of 2014, and an excess above baseline was first observed in England, the Netherlands and Portugal (week 50). As of week 9 2015, the mortality was still elevated in five of the 13 countries that were affected in consistent patterns of excess mortality in the elderly. Significant

\section{TABLE}

Excess mortality $\geq 2$ z-scores over baseline among individuals aged $\geq 65$ years and assessment of transmission intensity of influenza-like-illness in EuroMOMO countries, by week, week 49, 2014-week 9, 2015 ( $\mathrm{n}=16$ countries)

\begin{tabular}{|c|c|c|c|c|c|c|c|c|c|c|c|c|c|}
\hline \multirow{3}{*}{ Country } & \multicolumn{13}{|c|}{ Year - Week } \\
\hline & \multicolumn{4}{|c|}{2014} & \multicolumn{9}{|c|}{2015} \\
\hline & 49 & 50 & 51 & 52 & 1 & 2 & 3 & 4 & 5 & 6 & 7 & 8 & 9 \\
\hline Belgium & Low & Low & Low & Low & Low & Low & Medium & High & High & High & High & High & Medium \\
\hline Denmark & Low & Low & Low & Low & Low & Low & Low & Low & Low & Low & Low & Low & Low \\
\hline Estonia & Low & Low & Low & Low & Low & Low & Low & Low & Low & Medium & Medium & Medium & Medium \\
\hline France & Low & Low & Low & Low & Low & Medium & Medium & Medium & Medium & Medium & High & High & Medium \\
\hline Greece $^{b}$ & Low & Low & Low & Low & Low & Medium & Medium & High & Medium & Medium & Medium & & \\
\hline Hungary & Low & Low & Low & Low & Low & Low & Low & Medium & High & High & High & High & High \\
\hline The Netherlands & Low & Medium & Medium & Medium & Medium & Medium & Medium & Medium & Medium & Medium & Medium & Medium & Medium \\
\hline Portugal & Low & Low & Low & Low & Medium & Medium & Medium & High & Medium & Medium & Medium & Medium & Low \\
\hline Spain & Low & Low & Low & Low & Low & Medium & Medium & Medium & High & Medium & Medium & Medium & Medium \\
\hline Switzerland & Low & Low & Low & Low & Low & Medium & Medium & Medium & High & High & High & High & High \\
\hline UK-England & Low & Low & Medium & Medium & Medium & Medium & Medium & Medium & Medium & Low & Low & Low & Low \\
\hline UK-Northern Ireland & Low & Low & Low & Low & Low & Medium & Medium & Medium & Medium & Medium & Medium & Medium & Medium \\
\hline UK-Scotland & Low & Low & Low & Low & Medium & Medium & Medium & Medium & Medium & Medium & Medium & Medium & Medium \\
\hline UK-Wales & Low & Low & Low & Low & Low & No info & Low & Low & Low & No info & Low & No info & No info \\
\hline
\end{tabular}

Excess mortality ( $\geq 2$ z-scores over baseline) among individuals aged $\geq 65$ years

EuroMOMO: European monitoring of excess mortality for public health action; UK: United Kingdom; z-score: standardised deviation from the baseline.

The terms high, medium and low in the cells of the table refer to the intensity of influenza-like illness transmission.

a Corresponding to 1 December 2014-1 March 2015.

b Mortality data is missing in week 8 and 9, 2015. Mortality data is derived from municipalities of Athens, Keratsini-Drapetsona and Pireas as well as prefectures of Magnisia, Sporades, Kerkira, Achaia, Kavala and Thasos. 


\section{FIGURE 1}

Number of deaths by week and modelled baseline obtained from pooled analysis of data from EuroMOMO countries, week 23, 2010-week 9, 2015 ( $\mathrm{n}=14$ countries)

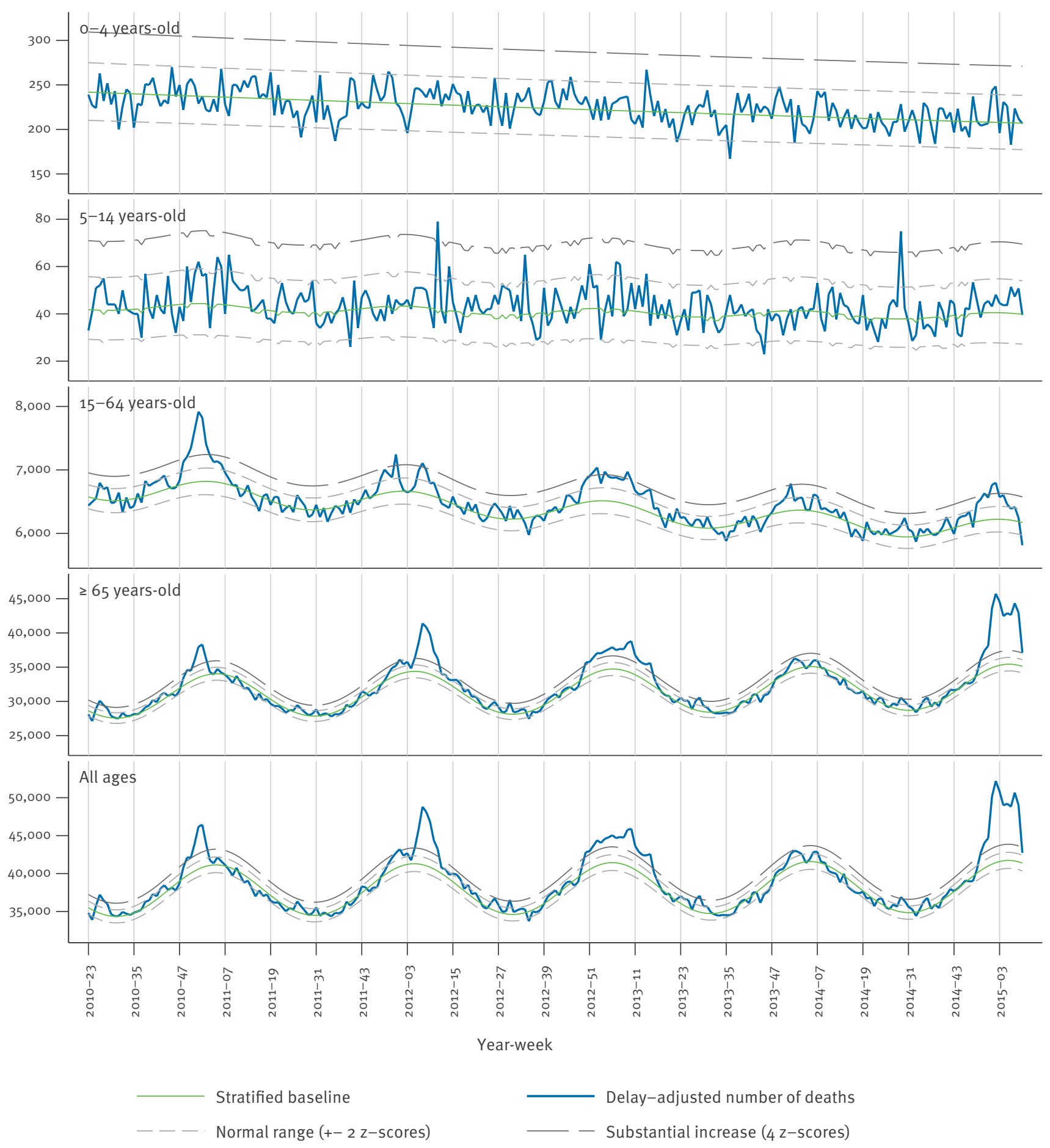

EuroMOMO: European monitoring of excess mortality for public health action; UK: United Kingdom; z-score: standardised deviation from the baseline.

Participating countries: Belgium, Denmark, Estonia, Finland, France, Hungary, Netherlands, Portugal, Spain, Sweden, Switzerland, UK (England), UK (Scotland), UK (Wales).

a Corresponding to 7 June 2010-1 March 2015. 
excess mortality was not yet observed in Estonia and Finland, and although excess mortality was recorded in Denmark this has, at present, only occurred over a single week. The Table describes the weekly patterns of excess mortality in relation to the assessment of intensity of influenza transmission as reported by ECDC.

The pooled analysis, which shows excess mortality across 14 participating countries combined, revealed a sharp rise in mortality among individuals aged $\geq 65$ years across Europe starting from week 49, 2014, crossing four z-scores above baseline in week 50, and reaching 21 z-scores in week 2 of 2015 (Figure 1). Some excess mortality was also observed in the age group 15-64 years, reaching four z-scores in week 51, 2014. In younger individuals, mortality has been within the expected range, although among children $<5$ years, z-scores slightly over 2 were reached in week 1 and 2, 2015 .

Figure 2 shows the excess mortality among the elderly (estimated from the pooled model expressed as the number of z-scores above the baseline) in the 14 countries included and the proportion positive for influenza detections in the European sentinel surveillance schemes. By visual inspection, the increase in excess mortality follows the same pattern as the increase in per cent positive influenza virus detections. Both start to increase around week 49 of 2014 but influenza per cent-positive reaches a plateau around week 4 to 8 while excess mortality seems to peak in week 2, 2015, but remains elevated at least until week 8 . Of note, the reduction of mortality after week 8 still does not represent a decrease below the 2 z-scores.

\section{Discussion}

We found evidence of a significant increase in excess all-cause mortality predominantly in the elderly in 13 of the 16 countries examined. The weekly patterns of excess mortality in relation to the intensity of influenza transmission show that in most weeks excess mortality coincides with medium or high influenza activity (78 of 97 weeks with excess mortality).

The current influenza season in the northern hemisphere has been predominated by influenza $\mathrm{A}\left(\mathrm{H}_{3} \mathrm{~N}_{2}\right)$ virus [8], which according to FluNews Europe accounted for $56 \%$ of the detections across European Community

\section{FIGURE 2}

Excess mortality among those aged $\geq 65$ years, expressed as the number of $\mathrm{z}$-scores above the baseline (left axis) and the proportion positive for influenza detections in the European sentinel surveillance schemes (right axis), week 40, 2014week $9,2015^{\text {a }}$

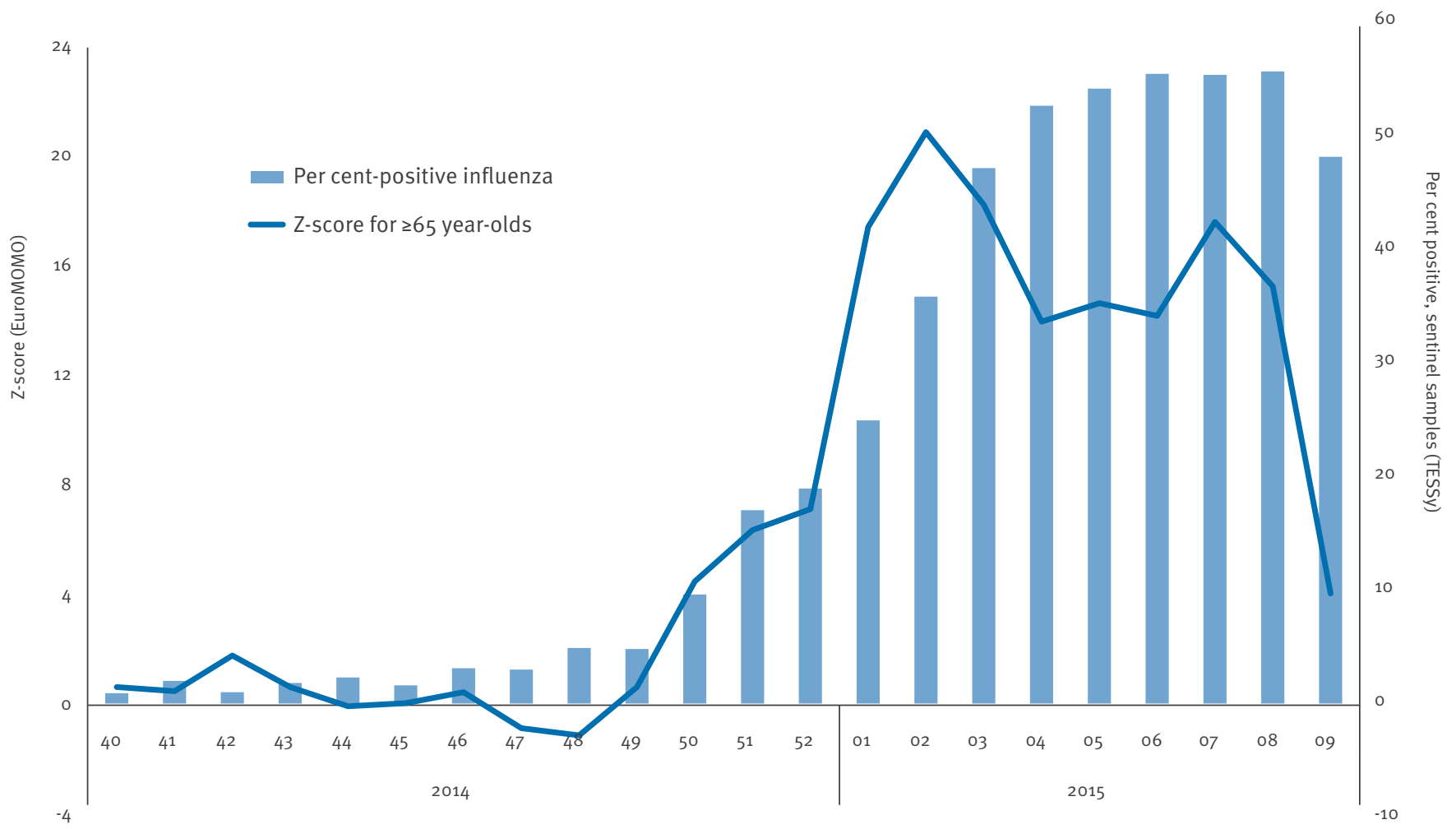

Year-week

EuroMOMO: European monitoring of excess mortality for public health action; TESSY: The European Surveillance System; UK: United Kingdom; z-score: standardised deviation from the baseline.

Participating countries for mortality data include Belgium, Denmark, Estonia, Finland, France, Hungary, the Netherlands, Portugal, Spain, Sweden, Switzerland and the UK (England, Wales, and Scotland)

a Corresponding to 29 September 2014-1 March 2015. 
(EC)/ European Economic Area (EEA)/WHO European region countries [7]. It is expected that a winter season with predominance of influenza $\mathrm{A}\left(\mathrm{H}_{3} \mathrm{~N}_{2}\right)$ has higher mortality impact on the elderly than a season with predominant influenza $\mathrm{A}\left(\mathrm{H}_{1} \mathrm{~N}_{1}\right)$ or a season with low influenza $A$ transmission $[1,2]$. Since its appearance in 2009, influenza $A\left(\mathrm{H}_{1} \mathrm{~N}_{1}\right)$ pdmog has been a prevailing virus type, and this type is known to have less impact on the elderly [1,2,9-11]. Furthermore, in the present winter season $2014 / 15$, most of the influenza $A\left(\mathrm{H}_{3} \mathrm{~N}_{2}\right)$ viruses characterised in Europe until week 9, 2015 exhibit antigenic differences from the virus included in the 2014/15 northern hemisphere influenza vaccine [5-8]. A reduction in the effectiveness of the $A\left(\mathrm{H}_{3} \mathrm{~N}_{2}\right)$ component of the influenza vaccine has been reported from several countries [12-15], which in consequence may lead to higher morbidity and mortality in vaccinated populations.

There are inconsistencies in the mortality findings, e.g. Finland and Sweden had several weeks of medium intensity of influenza transmission but until 1 March 2015 only two consecutive weeks with significant excess mortality were noted in Sweden and none in Finland. Furthermore, some countries see a pattern of an early rise in mortality whereas increases in influenza transmission (as assessed in the primary care sector) seems to appear later; this pattern, however is not unusual as will be discussed below.

There are several possible explanations for these observations. Firstly, an early rise in mortality may suggest that other factors, besides influenza, contribute to the occurrence of excess mortality. Temperature data from this winter indicate cold snaps in France, Greece, Spain and Switzerland (data not shown, available from the authors). Other infections, including respiratory syncytial virus (RSV)-associated disease might further add to excess mortality in some countries, but not in others. For example, the French syndromic surveillance systems noted intense activities for pneumonia and bronchitis starting from the end of 2014 (A. Fouillet, personal communication February 2015). Secondly, the assessment of influenza transmission intensity using primary care surveillance data is qualitative and is based on the available information and thus reflects the specific attributes of national surveillance systems, such as the population under surveillance. Influenza-like illness (ILI) consultations in primary care should be compared cautiously between countries, and although work has attempted to standardise this measure, ILI remains a concept to be treated with care, as it may not fully capture intensity of transmission $[16,17]$. Finally there may be a range of influenza-related factors such as differences in vaccination uptake; the effectiveness of the available vaccines against seasonal influenza or differences in circulating viruses and the extent of drift.

The pooled analyses of excess mortality result in a pattern that appears consistent with the European virological influenza data. The two time series follow a parallel increase, with an apparent stabilisation of the virological data from week 4,2015 . The interval from week 50, 2014, to week 3, 2015 when per centpositive increases is likely to represent the time-period with most intense circulation of influenza virus, and it is at the end of this ascent that the impact on mortality is expected to be highest. The apparent decrease in excess mortality at the end of the observation period needs to be treated with caution as it may be affected by an uncertainty in the adjusted delays in reporting of deaths.

The similar temporal pattern between the increase in per cent positive influenza virus detections and excess mortality is not a proof of causality. Nonetheless, it indicates that it is likely that influenza is an important contributor to the observed excess mortality among the elderly given previous evidence of attribution $[2,9,10,18,19]$. Pooling of data, in this case both data on number of deaths and virological influenza data across Europe, provides more statistical power than country-specific analyses and may enhance patterns that are not apparent in individual countries or populations [4]. On the other hand, details may be lost when pooling data (e.g. excess in an individual country can be diluted when others experience no excess) and therefore the two approaches of data analysis and visualisation are complementary.

Studies have shown that multivariable regression models can be successfully used to estimate the impact of mortality risks from influenza viruses while adjusting for the impact of other determinants including extreme temperature $[1,2,9,18,19]$. In order to assess the public health impact of influenza at the population level, we are working to develop a common European approach to estimate the number of excess deaths associated with influenza adjusting for other such factors, as discussed previously [3].

The influenza season in 2014/15 differs from the two past seasons across Europe. The past seasons showed a mixed and heterogeneous pattern of influenza $A\left(\mathrm{H}_{1} \mathrm{~N}_{1}\right)$ pdmo9, influenza $\mathrm{A}\left(\mathrm{H}_{3} \mathrm{~N}_{2}\right)$ and influenza $B$ with inter-country differences in the dominant circulating sub-type. Although the current season is not dominated entirely by influenza $\mathrm{A}\left(\mathrm{H}_{3} \mathrm{~N}_{2}\right)$ across Europe, this influenza type is certainly more commonly detected compared with the previous two seasons. It is well recognised that $A\left(\mathrm{H}_{3} \mathrm{~N}_{2}\right)$ tends to particularly impact the elderly (as was seen in seasons before the 2009 pandemic). In the current winter, the effect of this virus may possibly be exacerbated by the emergence of a drift variant. Furthermore, the available vaccine may not effectively cover this subtype. We hypothesise that the observed excess mortality among the elderly is a result of this epidemiological situation, potentially aggravated by the added effects of cold snaps and other respiratory infections. Further work is planned at the end of the season to investigate this hypothesis. 


\section{Acknowledgments}

The EuroMOMO network has received financial support from DG Sanco (Directorate-General for Health and Consumer Protection) through an operating grant $2013 \quad 3102 \quad$ SSI FY2014. We acknowledge all EuroMOMO partners for their contributions as well as the various National Offices of Statistics that are essential partners in setting up mortality monitoring.

\section{Conflict of interest}

None declared.

\section{Authors' contributions}

KM designed and wrote the first version of the manuscript. LE did the analyses, graphs and figures. AM coordinated all correspondences between authors and implemented most comments. KM, JN and LE implemented additional comments from authors. All authors provided data and contributed in the writing of, and approved the final version of the manuscript.

\section{References}

1. Matias G, Taylor R, Haguinet F, Schuck-Paim C, Lustig R, Shinde V. Estimates of mortality attributable to influenza and RSV in the United States during 1997-2009 by influenza type or subtype, age, cause of death, and risk status. Influenza Other Respi Viruses. 2014;8(5):507-15. http://dx.doi.org/10.1111/ irv.12258 PMID:24975705

2. Nielsen J, Mazick A, Glismann S, Mølbak K. Excess mortality related to seasonal influenza and extreme temperatures in Denmark, 1994-2010. BMC Infect Dis. 2011;11(1):350. http:// dx.doi.org/10.1186/1471-2334-11-350 PMID:22176601

3. Mazick A, Gergonne B, Nielsen J, Wuillaume F, Virtanen MJ, Fouillet $A$, et al. Excess mortality among the elderly in 12 European countries, February and March 2012. Euro Surveill. 2012;17(14):20138. PMID:22516003

4. Nielsen J, Mazick A, Andrews N, Detsis M, Fenech TM, Flores VM, et al. Pooling European all-cause mortality: methodology and findings for the seasons 2008/2009 to 2010/2011. Epidemiol Infect. 2013;141(9):1996-2010. http://dx.doi. org/10.1017/So950268812002580 PMID:23182146

5. European Centre for Disease Prevention and Control (ECDC). Influenza activity maps for Europe. Stockholm: ECDC. [Accessed 13 March 2015]. Available from: http://ecdc.europa. eu/en/healthtopics/seasonal_influenza/Pages/index.aspx

6. European Centre for Disease Prevention and Control (ECDC). Influenza virus characterisation, summary Europe, December 2014. Stockholm: ECDC; 2015. Available from: http://ecdc. europa.eu/en/publications/Publications/influenza-viruscharacterisation-December-2014.pdf

7. FluNews Europe. Joint ECDC / WHO Europe weekly influenza update bulletin. ECDC/WHO. [Accessed 13 March 2015]. Available from: http://www.flunewseurope.org/

8. van der Werf S, Lévy-Bruhl D. Influenza - the need to stay ahead of the virus. Euro Surveill. 2015;20(5):21030. http:// dx.doi.org/10.2807/1560-7917.ES2015.20.5.21030. Available from: http://www.eurosurveillance.org/ViewArticle. aspx?Articleld=21030 PMID:25677049

9. Hardelid P, Pebody R, Andrews N. Mortality caused by influenza and respiratory syncytial virus by age group in England and Wales 1999-2010. Influenza Other Respi Viruses. 2013;7(1):35-45. http://dx.doi.org/10.1111/j.17502659.2012.00345.x PMID:22405488

10. Wijngaard CC, Asten L, Koopmans MP, Pelt W, Nagelkerke NJ, Wielders CC, et al. Comparing pandemic to seasonal influenza mortality: moderate impact overall but high mortality in young children. PLoS ONE. 2012;7(2):e31197. http://dx.doi. org/10.1371/journal.pone.0031197 PMID:22319616

11. Viboud C, Miller M, Olson D, Osterholm M, Simonsen L. Preliminary estimates of mortality and years of life lost associated with the $2009 \mathrm{~A} / \mathrm{H}_{1} \mathrm{~N}_{1}$ pandemic in the US and comparison with past influenza seasons. PLoS Curr. 2010;2:RRN1153. http://dx.doi.org/10.1371/currents.RRN1153 PMID:20352125
12. Skowronski D, Chambers C, Sabaiduc S, De Serres G, Dickinson J, Winter A, et al. Interim estimates of 2014/15 vaccine effectiveness against influenza $\mathrm{A}\left(\mathrm{H}_{3} \mathrm{~N}_{2}\right)$ from Canada s Sentinel Physician Surveillance Network, January 2015. Euro Surveill. 2015;20(4):21022. http://dx.doi.org/10.2807/15607917.ES2015.20.4.21022 PMID:25655053

13. McNeil S, Andrew M, Ye L, Haguinet F, Hatchette T, ElSherif $M$, et al.; Investigators of the Serious Outcomes Surveillance Network of the Canadian Immunization Research Network (CIRN). Interim estimates of 2014/15 influenza vaccine effectiveness in preventing laboratory-confirmed influenzarelated hospitalisation from the Serious Outcomes Surveillance Network of the Canadian Immunization Research Network, January 2015. Euro Surveill. 2015;20(5):21024. http://dx.doi. org/10.2807/1560-7917.ES2015.20.5.21024 PMID:25677052

14. Pebody R, Warburton F, Ellis J, Andrews N, Thompson C, von Wissmann $B$, et al. Low effectiveness of seasonal influenza vaccine in preventing laboratory-confirmed influenza in primary care in the United Kingdom: 2014/15 mid-season results. Euro Surveill. 2015;20(5):21025. http://dx.doi. org/10.2807/1560-7917.ES2015.20.5.21025 PMID:25677050

15. Flannery B, Clippard J, Zimmerman RK, Nowalk MP, Jackson ML, Jackson LA, et al.; Centers for Disease Control and Prevention. Early estimates of seasonal influenza vaccine effectiveness - United States, January 2015. MMWR Morb Mortal Wkly Rep. 2015;64(1):10-5. PMID:25590680

16. Vega T, Lozano JE, Meerhoff T, Snacken R, Mott J, Ortiz de Lejarazu R, et al. Influenza surveillance in Europe: establishing epidemic thresholds by the moving epidemic method. Influenza Other Respi Viruses. 2013;7(4):546-58. http://dx.doi. org/10.1111/j.1750-2659.2012.00422.x PMID:22897919

17. Green HK, Charlett A, Moran-Gilad J, Fleming D, Durnall $\mathrm{H}$, Thomas DR, et al. Harmonizing influenza primary-care surveillance in the United Kingdom: piloting two methods to assess the timing and intensity of the seasonal epidemic across several general practice-based surveillance schemes. Epidemiol Infect. 2015;143(1):1-12. http://dx.doi.org/10.1017/ S0950268814001757 PMID:25023603

18. Van Asten L, van den Wijngaard C, van Pelt W, van de Kassteele J, Meijer A, van der Hoek W, et al. Mortality attributable to 9 common infections: Significant effect of influenza A, RSV, influenza $B$, norovirus and parainfluenza in the elderly. J Infect Dis. 2012;206:628-39. http://dx.doi.org/10.1093/infdis/jis415 PMID:22723641

19. Cox B, Wuillaume F, Van Oyen H, Maes S. Monitoring of allcause mortality in Belgium (Be-MOMO): a new and automated system for the early detection and quantification of the mortality impact of public health events. Int J Public Health. 2010;55(4):251-9. http://dx.doi.org/10.1007/s00038-010-01356 PMID:20376690 